\title{
PESQUISA DE TENDÊNCIAS: investigação de referenciais para o projeto de móveis
}

\author{
Zamoner, Michele Tais D. C.; E; Universidade Federal do Paraná; \\ mizamoner@gmail.com \\ Razera, Dalton; Dr.; Universidade Federal do Paraná; \\ daltonrazera@ufpr.br \\ Heemann, Adriano; Dr.; Universidade Federal do Paraná; \\ adriano.heemann@gmail.com \\ Barauna, Débora; MSc; Universidade Federal do Paraná; \\ dbarauna@univille.br
}

\begin{abstract}
Resumo: Este artigo apresenta uma investigação referente às principais fontes de informação relevantes para a pesquisa de tendências em projeto de mobiliário no Brasil. $O$ estudo foi realizado por meio de uma revisão de literatura sistematizada, ponderando os trabalhos publicados nos últimos 10 anos. $O$ resultado alcançado contempla o mapeamento dos principais autores e fontes de informações que abordam aspectos ligados à sustentabilidade e ao ecodesign como tendências para a indústria moveleira. Considera-se que a identificação dos conteúdos informativos determinados possa auxiliar o designer e demais profissionais da indústria moveleira na elaboração de produtos inovadores, competitivos e alinhados às necessidades do mercado.
\end{abstract}

Palavras-chave: pesquisa de tendências, sustentabilidade, mobiliário, painel de tendências.

Abstract: This paper article regards a major research including the main information sources of the trends for the Brazilian market on furniture. The investigation method consisted on reviewing a ten years period of systematic literature published. The results achieved include mapping the main authors and information sources related to pointing ecodesign and sustainability as the most natural tendencies for the furniture industry. The delimitation and identification of informative content might support the designer and other professionals in the furniture industry in developing innovative and competitive products, aligned with market needs.

Keywords: research trends, sustainability, furniture, trends panel. 


\title{
1. INTRODUÇÃO
}

A pesquisa de tendências visa sinalizar como as constantes transformações sociais, culturais e políticas influenciam diretamente a vida das pessoas moldando suas preferências de consumo.

\begin{abstract}
A cada ano, novas formas, cores, materiais e funções configuram o mobiliário comercializado no Brasil. Essas mudanças obedecem a um ciclo e adquirem uma importância especial na vida das pessoas que passam a seguir estes padrões e considerar menos importante o que não pertence a esse conjunto. (DE SOUZA, 2009, p.17).
\end{abstract}

Com base nessa afirmação, o objetivo deste estudo foi identificar os principais autores e fontes de informações que desenvolvem pesquisas de tendências como ferramenta de inovação para o desenvolvimento de novos produtos, tendo como motivação os aspectos ligados à sustentabilidade e ao ecodesign para o mercado moveleiro.

O desdobramento deste estudo está dividido em 3 seções. A primeira seção é a introdução, a segunda seção é constituída pela metodologia, onde abordará de forma descritiva as ferramentas e métodos utilizados para alcançar o objetivo desejado. A terceira seção, é constituída de uma fundamentação teórica, quadros informativos desenvolvido pelos autores e figuras que servirão para exemplificar e discutir os resultados encontrados na pesquisa.

O resultado deste estudo contempla no mapeamento das principais referências em pesquisa de tendências para o projeto de móveis. Essas referências abordam os conteúdos publicados no meio acadêmico por meio de artigos, dissertações e teses e, os conteúdos documentais analisados em sites, blogs, feiras, livros e cartilhas. A aplicabilidade deste estudo permite o conhecimento atualizado e a identificação de lacunas que possam auxiliar o trabalho do designer e do profissional da indústria moveleira na elaboração de novos produtos.

\section{MÉTODO}

A investigação foi organizada com base na Revisão Bibliográfica Sistemática (RBS), que orientou a seleção dos conteúdos publicados nos últimos 10 anos.

A pesquisa consolidou as informações identificadas através do portal de busca da Coordenação de Aperfeiçoamento de Pessoal de Nível Superior (CAPES ${ }^{1}$ ), e do Google ${ }^{2}$ (2014). Para a estratégia de busca foram submetidas palavras-chaves ou strings nas bases de dados eletrônicas. Os termos relacionados para submissão as fontes são apresentados a seguir: "pesquisa de tendências AND mobiliário AND móveis", "sustentabilidade AND novas tecnologias AND novos materiais" e, que através do resultado obtido foi possível identificar, selecionar e analisar os estudos incluídos na revisão.

\footnotetext{
${ }^{1}$ http://www.periodicos.capes.gov.br/

${ }^{2}$ http://scholar.google.com.br/
} 
A RBS constitui-se principalmente de materiais elaborados e revisados por pares; teses, dissertações e artigos, tendo como o idioma definido para a pesquisa, conteúdos disponibilizados em português .

Considerou-se relevante uma pesquisa documental com materiais que ainda não receberam tratamento analítico, como resultados foram levantados alguns institutos de pesquisas de tendências (nacionais e internacionais), cartilhas, cadernos ou referenciais de tendências, materiais de feiras (nacionais e internacionais), sites, blogs, estudos realizados por entidades, sindicatos e empresas, relacionados ao tema, tais como SEBRAE ${ }^{3}$, SENAI $^{4}$, SINDUSMÓBIL ${ }^{5}$, SHATTDECOR ${ }^{6}$.

A partir desses conteúdos encontrados, foi realizado o mapeamento dos principais autores, referenciais, fontes de informações, e analisou-se qualitativamente os métodos e ferramentas encontrados.

\title{
3. RESULTADOS E DISCUSSÃO
}

As palavras moda e tendência não devem ser confundidas, "moda" significa, no cotidiano, ser "muito usado", já a palavra "tendência" vem do latim tendentia, proveniente do verbo tendere, que tem por significado "tender para", "inclinar-se para" ou "ser atraído por". Assim, tendência é uma manifestação, na esfera do comportamento, do consumo ou do "espírito do tempo", de uma sensibilidade anunciada por sinais (JAYME, 2009). Neste sentido, afirma-se que:

\begin{abstract}
Empresas de sucesso devem praticar a observação de tendências e o planejamento de cenários. Nunca será prejudicial identificar tendências e especular sobre suas implicações para a empresa. As empresas também podem se beneficiar imaginando diferentes cenários futuros e planejando respostas para eles. Empresas inteligentes poderão nomear uma pessoa ou um grupo para monitorar tendências e testar cenários. (KOTLHER, 2005, apud JAYME, 2009).
\end{abstract}

Assim, as tendências tem grande importância na construção de cenários, sendo fonte de interpretação para a realização de projeções sobre o futuro das empresas. Ainda segundo Jayme (2009), a maioria dos institutos de pesquisa de tendências é formado por organizações que atuam de forma globalizada e com fins lucrativos, e para se obter informações é necessário ser filiado. Estes institutos estão localizados em grandes centros urbanos, com escritórios em diferentes continentes e com clientes e colaboradores em todo o mundo. Trabalham identificando os mais variados tipos de tendências de uma região, ou de um mercado específico, eles buscam prever e entender as motivações e o comportamento do mercado consumidor, além de estabelecer os prováveis cenários onde as empresas irão atuar.

\footnotetext{
${ }^{3}$ Serviço Brasileiro de Apoio às Micro e Pequenas Empresas.

${ }^{4}$ Serviço Nacional de Aprendizagem Nacional.

${ }^{5}$ Sindicato Das Indústrias da Construção e do Mobiliário de São Bento Do Sul.

${ }^{6}$ Fabricante Alemã de papéis decorativos impressos com sede no Brasil na cidade de São José dos Pinhais, Paraná.
} 
Com base na dissertação de De Souza (2009), vários termos são encontrados para designar e classificar as tendências. Para facilitar a compreensão, a Figura 1, ilustra a comparação entre esses termos.

\begin{tabular}{|c|c|}
\hline CLASSIFICAÇAO & SENTIDO \\
\hline Metatendência & $\begin{array}{l}\text { Origem de todas as categorias de } \\
\text { tendências. }\end{array}$ \\
\hline \multicolumn{2}{|l|}{ Macrotendência|Tendência } \\
\hline de Fundo & $\begin{array}{l}\text { O tipo de tendência que influencia o } \\
\text { consumidor por longos períodos de } \\
\text { tempo. Derivadas de fatores globais } \\
\text { que influenciam diretamente a vida das } \\
\text { pessoas. }\end{array}$ \\
\hline $\begin{array}{c}\text { Microtendência / Tendência } \\
\text { de Ciclo Curto }\end{array}$ & $\begin{array}{l}\text { Fenômenos passageiros, de ordem } \\
\text { estética, que promovem o consumismo. } \\
\text { Podem ser consideradas como } \\
\text { modismos. }\end{array}$ \\
\hline Contratendência & $\begin{array}{l}\text { Fenômeno de sentido oposto, porém de } \\
\text { mesma procedência das tendências. } \\
\text { Elas formam um par positivo ou } \\
\text { negativo com as tendências. }\end{array}$ \\
\hline
\end{tabular}

Figura 1 - Classificação das tendências

Fonte: De Souza (2009,p.31).

A seguir, nos Quadros 1 e 2 são apresentados os institutos de pesquisa nacionais e internacionais, possíveis de fornecer informações sobre macrotendências (tecnologias, economias, sociais, demográficas, ambientais, culturais).

Quadro 1 : Estudo analítico dos Institutos de pesquisa de tendências.

\begin{tabular}{|l|l|l|}
\hline Instituto/ Local & Ferramentas & Profissionais Envolvidos \\
\hline $\begin{array}{l}\text { CIFS-Copenhagen } \\
\text { Institute for } \\
\begin{array}{l}\text { Futures Studies } \\
\text { (Copenhagen) }\end{array}\end{array}$ & $\begin{array}{l}\text { Análises, estatísticas, identificação de } \\
\text { tendências globais e de megatendências. }\end{array}$ & $\begin{array}{l}\text { Acadêmicos, Profissionais em } \\
\text { economia, ciência política, } \\
\text { etnologia, geografia cultural, } \\
\text { astrofísica, direito, filosofia, } \\
\text { psicologia, religião. }\end{array}$ \\
\hline DEMOS - Londres & $\begin{array}{l}\text { Análise de cenários através de áreas chaves, } \\
\text { demografia, economia, tecnologia e } \\
\text { capacidade de inovação. }\end{array}$ & $\begin{array}{l}\text { Serviços públicos, ciência e } \\
\text { tecnologia, artes, identidade e } \\
\text { cultura, cidades e espaço, } \\
\text { público, segurança global. }\end{array}$ \\
\hline $\begin{array}{l}\text { Future Concept Lab } \\
\text { - Milão }\end{array}$ & $\begin{array}{l}\text { Pesquisa qualitativa, Pesquisa quantitativa, } \\
\text { Estudos sociológicos, semióticos e } \\
\text { antropológicos. Pesquisa etnográfica Pesquisa } \\
\text { sócio-cultural, Estilos mentais, Sinais de rua, } \\
\text { Programa Felicidade. }\end{array}$ & $\begin{array}{l}\text { Semiótica, sociologia, } \\
\text { antropologia, etnografia, design, } \\
\text { moda, ciências Sociais, } \\
\text { comunicação, marketing } \\
\text { Sistemas de Informação, } \\
\text { economia. }\end{array}$ \\
\hline
\end{tabular}

Fonte: Elaborado pelos autores, com base na pesquisa realizada - continuação. 
Quadro 2 : Estudo analítico dos Institutos de pesquisa de tendências - continuação.

\begin{tabular}{|c|c|c|}
\hline $\begin{array}{l}\text { Future Foundation } \\
\text { - Londres }\end{array}$ & $\begin{array}{l}\text { Técnica de cenários, pesquisas quantitativas, } \\
\text { qualitativas, matemática e técnicas estatísticas } \\
\text { a fim de mapear tendências chaves e fornecer } \\
\text { acesso ilimitado a relatórios. }\end{array}$ & $\begin{array}{l}\text { Pesquisa de Marketing, } \\
\text { econômica e social, } \\
\text { conhecimento em tendências } \\
\text { sócio econômicas, ciências } \\
\text { sociais, matemática e } \\
\text { estatística. }\end{array}$ \\
\hline $\begin{array}{l}\text { GBN - Global } \\
\text { Business Network - } \\
\text { São Francisco- } \\
\text { Califórnia }\end{array}$ & $\begin{array}{l}\text { Técnicas quantitativas, qualitativas, } \\
\text { matemáticas e estatísticas, estuda demografia, } \\
\text { forças sociais, geopolíticas, ciência e } \\
\text { tecnologia. }\end{array}$ & $\begin{array}{l}\text { Administração, economia, artes, } \\
\text { arquitetura, física, literatura, } \\
\text { tecnologia, sociologia entre } \\
\text { outros. }\end{array}$ \\
\hline $\begin{array}{l}\text { Henley Centre - } \\
\text { Londres, New York } \\
\text { e India. }\end{array}$ & $\begin{array}{l}\text { Técnicas de Cenário e futuro, técnica de } \\
\text { modelagem dos dados, dados qualitativos, } \\
\text { quantitativos, pesquisa etnográfica, } \\
\text { fotografias, vídeos, estilo de vida. }\end{array}$ & $\begin{array}{l}\text { Consultores estratégicos, } \\
\text { projetistas, peritos qualitativos } \\
\text { e quantitativos e especialistas } \\
\text { em comercialização, marketing } \\
\text { e publicidade. }\end{array}$ \\
\hline $\begin{array}{l}\text { NEF - Núcleo de } \\
\text { Estudos do Futuro - } \\
\text { PUC - São Paulo }\end{array}$ & $\begin{array}{l}\text { Técnicas de amostragem a análises } \\
\text { estatísticas, coleta de dados, pesquisas de } \\
\text { opinião, técnicas participativas. Descrevem e } \\
\text { analisam as tendências passadas e condições } \\
\text { do presente para projetar e construir o futuro. }\end{array}$ & Não apresenta. \\
\hline $\begin{array}{l}\text { OBSERVATÓRIO de } \\
\text { SINAIS - São Paulo }\end{array}$ & $\begin{array}{l}\text { Análise transversal, imersão na pesquisa, } \\
\text { monitoração de vetores, pontos de venda, } \\
\text { mapas de tendências. }\end{array}$ & $\begin{array}{l}\text { Sociologia, antropologia, } \\
\text { arquitetura, artes, moda, } \\
\text { design. }\end{array}$ \\
\hline $\begin{array}{l}\text { SENAI / CETIQT - } \\
\text { Rio de Janeiro. }\end{array}$ & $\begin{array}{l}\text { Estudos de cor, tendências de moda e design, } \\
\text { prospecção tecnológica e mercadológica. }\end{array}$ & Não apresenta. \\
\hline $\begin{array}{l}\text { RISC } \\
\text { INTERNATIONAL - } \\
\text { Paris e Suíça }\end{array}$ & $\begin{array}{l}\text { Surveys quantitativas, Pesquisa em ciências } \\
\text { sociais, dados qualitativos. }\end{array}$ & $\begin{array}{l}\text { Marketing, antropologia, } \\
\text { sociologia, vendas, matemática, } \\
\text { estatística, economia, finanças, } \\
\text { publicidade, arte, tecnologia, } \\
\text { línguas, ensino e administração. }\end{array}$ \\
\hline $\begin{array}{l}\text { IFTF -The Institute } \\
\text { for the Future - } \\
\text { Palo Alto California }\end{array}$ & $\begin{array}{l}\text { Pesquisa quantitativa, etnográfica, observação } \\
\text { direta oficinas com especialistas visitas a } \\
\text { laboratórios e universidades seminários e } \\
\text { entrevistas especializadas, cenários, } \\
\text { modelagem, mapeamento. }\end{array}$ & $\begin{array}{l}\text { Tecnologia, saúde, sociologia, } \\
\text { economia, negócios } \\
\text { internacionais, design gráfico e } \\
\text { de jogos, sustentabilidade, } \\
\text { psicologia, antropologia. }\end{array}$ \\
\hline $\begin{array}{l}\text { WGSN -Worth } \\
\text { Global Style } \\
\text { Network - Londres, } \\
\text { New York, São } \\
\text { Paulo. }\end{array}$ & $\begin{array}{l}\text { Pesquisa sobre o mercado têxtil, investigação } \\
\text { de tendências de rua, fotografando como as } \\
\text { pessoas se vestem. Relatórios das principais } \\
\text { passarelas, locais preferidos dos jovens, } \\
\text { mercado, música, entretenimento e livrarias. }\end{array}$ & $\begin{array}{l}\text { Designers, pesquisadores, } \\
\text { jornalistas, fotógrafos. }\end{array}$ \\
\hline $\begin{array}{l}\text { Observatório de } \\
\text { Tendências del } \\
\text { Habitat - Valência } \\
\text { (Espanha) }\end{array}$ & $\begin{array}{l}\text { Perspectiva global sobre o habitat, definindo } \\
\text { tendência do morar. Conhecimento das causas } \\
\text { que impulsionam a tendência, identificação do } \\
\text { consumidor potencial e como se manifesta no } \\
\text { momento. Estudo de elementos da } \\
\text { arquitetura, design de interiores, mobiliário, } \\
\text { têxteis para o lar e revestimentos, abordagem } \\
\text { de estratégias de comunicação e distribuição } \\
\text { com o entorno sociocultural onde o habitat } \\
\text { está inserido. }\end{array}$ & $\begin{array}{l}\text { Design, arquitetura, sociologia } \\
\text { economia, marketing, } \\
\text { comunicação. }\end{array}$ \\
\hline
\end{tabular}

Fonte: Elaborado pelos autores, com base na pesquisa realizada. 
Segundo a Rede Design Brasil ${ }^{7}$, a fim de auxiliar os empresários e profissionais de design na concepção de produtos inovadores, algumas instituições publicam com periodicidade semestral ou anual os chamados Cadernos ou Referenciais de Tendências. No Brasil, através da iniciativa do SENAI-CETEMO, desde 2002 é elaborado anualmente um caderno de tendências para o segmento de móveis (Figura 2), com o objetivo de aproximar os conceitos dos materiais às necessidades dos empresários e designers, focados nos desejos e comportamento do consumidor final.

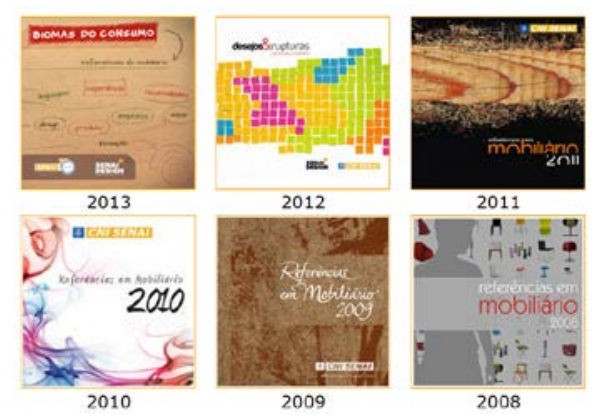

Figura 2 - Biomas do consumo - referências do mobiliário 2013.

Fonte: $\mathrm{CETEMO}^{8}$.

A empresa alemã SCHATTDECOR, anualmente divulga um livro de tendências decorativas desenvolvido pela equipe interna de design (Figura 3 ) e afirma que:

É preciso desenvolver um conceito de tendência exclusivo e independente, que forneça uma valiosa orientação e informações confiáveis a desenvolvedores de produtos, decoradores e designers. Para chegar a ele, são fundamentais as visitas às feiras, os workshops internos de design, pesquisas e análises. No entanto, apontar a cada ano tendências novas e absolutamente diversas não é mais condizente com o momento que vivemos. Tendências precisam de tempo para se desenvolver ou se confirmar. Morar continua sendo uma questão de gosto pessoal e, portanto, menos submetida às tendências de curto prazo ditadas pela moda. (TRENDS SCHATTDECOR, 2012/2013).

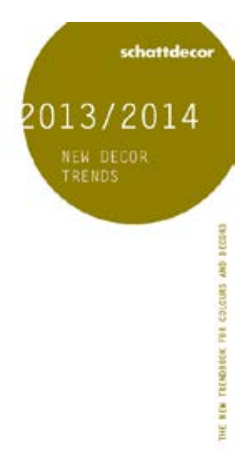

Figura 3 - Capa do Livro de tendências 2013/2014.

Fonte: SCHATTDECOR ${ }^{9}$ (2014).

\footnotetext{
${ }^{7}$ O portal Design Brasil é uma iniciativa do Ministério do Desenvolvimento, Indústria e Comércio Exterior (MDIC) com o apoio da Apex Brasil e coordenação do Centro Brasil Design. Disponível em: <http://www.designbrasil.org.br/quem-somos/> Acesso em Maio 2014.

${ }^{8}$ Disponível em: <http://www.cetemo.com.br/att design.html > Acesso em Abr. 2014

${ }^{9}$ Disponível em: <http://www.schattdecor.de/pt/padroes-tendencias/tendencias/> Acesso em Abr. 2014
} 
Disponibilizado pelo SINDUSMOBIL, a cartilha do BIOMOVEL $^{10}$ (Figura 4) apresenta a sustentabilidade como um caminho sem volta, afirma-se (BIOMÓVEL, 2009) que a conscientização sobre a preservação ambiental está no comportamento das pessoas, influenciando suas escolhas de consumo incluindo a compra de móveis e decorações.

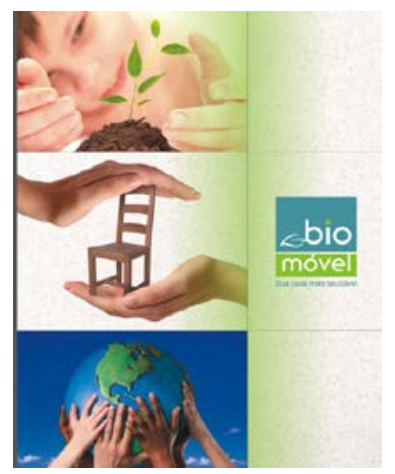

Figura 4 - Cartilha Biomóvel.

Fonte: SINDUSMOBIL (2009).

Através dos critérios definidos para a realização da RBS foram apontados 43 documentos publicados sobre o tema entre os anos de 2004 a 2014. Os documentos encontrados pelos sites de busca contém trabalhos disponibilizados em português das universidades do Brasil e de Portugal.

Com a finalidade de identificar os trabalhos acadêmicos publicados, o Quadro 3 apresenta as teses e dissertações encontrados na pesquisa.

Quadro 3: Lista de teses, dissertações selecionados para leitura.

\begin{tabular}{|l|l|l|l|l|}
\hline Elemento & \multicolumn{1}{|c|}{ Autor } & \multicolumn{1}{|c|}{ Título } & Local & Ano \\
\hline Tese & Liliane Iten Chaves. & $\begin{array}{l}\text { DESIGN POLIMI - IT } \\
\text { ENVIRONMENTAL SUSTAINABILITY: } \\
\text { design strategies, methods and tools } \\
\text { in the furniture sector }\end{array}$ & 2007 \\
\hline Dissertação & $\begin{array}{l}\text { Aline Teixeira de } \\
\text { Souza. }\end{array}$ & $\begin{array}{l}\text { DIRETRIZES PROJETUAIS E AS } \\
\text { TENDÊNCIAS: o design de móveis } \\
\text { residenciais no polo de arapongas. }\end{array}$ & UNESP & 2009 \\
\hline Dissertação & Maria Emilia & $\begin{array}{l}\text { TENDÊNCIAS: recomendações para } \\
\text { seu uso na gestão do design das } \\
\text { MPEs produtoras de louça de mesa } \\
\text { de Campo Largo -PR. }\end{array}$ & UFPR & 2009 \\
\hline Dissertação & $\begin{array}{l}\text { Glória Lucía } \\
\text { Rodríguez Correia } \\
\text { de Arruda }\end{array}$ & $\begin{array}{l}\text { O DESIGN NA INDÚSTRIA MOVELEIRA } \\
\text { BRASILEIRA E SEUS ASPECTOS } \\
\text { SUSTENTÁVEIS: } \\
\text { estudo de caso no pólo moveleiro de } \\
\text { Arapongas-Pr. }\end{array}$ & NESP & 2009 \\
\hline
\end{tabular}

Fonte: Elaborado pelos autores, com base na pesquisa realizada.

${ }^{10}$ Cartilha desenvolvida pelo Sindicato das Indústrias da Construção e do Mobiliário de São Bento do SulSC. Disponível em: <http:// www.sindusmobil.com.br/index.php/biomovel> Acesso em Abr. 2014. 
Já o Quadro 4, sintetiza a análise de artigos encontrados na RBS.

Quadro 4: Lista de artigos selecionados para leitura.

\begin{tabular}{|c|c|c|c|c|}
\hline Elemento & Autor & Título & Local & Ano \\
\hline Artigo & $\begin{array}{l}\text { Jucelia S. Giacomini } \\
\text { da Silva e } \\
\text { Aguinaldo dos } \\
\text { Santos }\end{array}$ & $\begin{array}{l}\text { IMPLICAÇÕES DOS CONCEITOS DA } \\
\text { SUSTENTABILIDADE NO DESIGN: uma } \\
\text { revisão crítica. }\end{array}$ & UFPR & 2009 \\
\hline Artigo & Liliane Iten Chaves. & $\begin{array}{l}\text { DESIGN PARA A SUSTENTABILIDADE } \\
\text { AMBIENTAL: estratégias, métodos e } \\
\text { ferramentas de design para o setor de } \\
\text { móveis. }\end{array}$ & UFPR & 2010 \\
\hline Artigo & Juliana Cardoso & $\begin{array}{l}\text { O DESIGN INDUSTRIAL COMO } \\
\text { FERRAMENTA PARA A } \\
\text { SUSTENTABILIDADE: estudo de caso do } \\
\text { couro de peixe. }\end{array}$ & UFU & 2010 \\
\hline Artigo & $\begin{array}{l}\text { Aline Teixeira de } \\
\text { Souza }\end{array}$ & $\begin{array}{l}\text { AS TENDÊNCIAS E O DESIGN: } \\
\text { metodologia de projeto do mobiliário } \\
\text { orientada para o futuro. }\end{array}$ & UNESP & 2010 \\
\hline Artigo & $\begin{array}{l}\text { Sandro Fábio } \\
\text { César, Rita Dione } \\
\text { Araújo Cunha, } \\
\text { João Paulo Leite } \\
\text { Guedes }\end{array}$ & $\begin{array}{l}\text { DESIGN, ARTE E SUSTENTABILIDADE: } \\
\text { Móveis A Partir Da Reciclagem De } \\
\text { Madeira. }\end{array}$ & UFBA & 2010 \\
\hline Artigo & $\begin{array}{l}\text { Marta Fabiano } \\
\text { Sambiase, } \\
\text { Marcos Antonio } \\
\text { Franklin, Jaqueline } \\
\text { Alfim Teixeira }\end{array}$ & $\begin{array}{l}\text { INOVAÇÃO PARA O } \\
\text { DESENVOLVIMENTO SUSTENTÁVEL } \\
\text { COMO FATOR DE COMPETITIVIDADE } \\
\text { PARA AS ORGANIZAÇÕES: Um Estudo } \\
\text { De Caso Duratex. }\end{array}$ & MACKENZIE & 2013 \\
\hline Artigo & $\begin{array}{l}\text { Lisiane Pellini Faller } \\
\text { Martinho Isnard } \\
\text { Ribeiro de Almeida }\end{array}$ & $\begin{array}{l}\text { PLANEJAMENTO POR CENÁRIOS: } \\
\text { preparando pequenas empresas do } \\
\text { varejo de móveis planejados para um } \\
\text { futuro competitivo. }\end{array}$ & UFSM/USP & 2014 \\
\hline
\end{tabular}

Fonte: Elaborado pelos autores, com base na pesquisa realizada.

$\mathrm{Na}$ pesquisa documental identificou-se fontes que poderão fornecer informações sobre as tendências de curto prazo, podendo influenciar 0 desenvolvimento de mobiliário, trazendo evidências sobre cores, formas, padrões, conceitos, combinações com componentes, outros produtos ou acessórios, novas tecnologias, materiais, etc.

Estes conteúdos foram agrupados por categorias, podendo ser visualizado nos Quadros 5 e 6.

Quadro 5: Fontes de informações para tendências em mobiliário.

\begin{tabular}{|l|l|l|}
\hline ELEMENTO & ITEM & EXEMPLO \\
\hline Seminários e & Nacionais e & Congresso moveleiro \\
Congressos & Internacionais & $\begin{array}{l}\text { Seminário moveleiro } \\
\text { Seminário internacional da indústria moveleira } \\
\end{array}$ \\
& & $\begin{array}{l}\text { P\&D Design } \\
\text { Simpósio de sustentabilidade }\end{array}$ \\
\hline
\end{tabular}

Fonte: Elaborado pelos autores, com base na pesquisa realizada - Continuação. 
Quadro 6: Fontes de informações para tendências em mobiliário - Continuação.

\begin{tabular}{|c|c|c|}
\hline Feiras setoriais & Nacionais & $\begin{array}{l}\text { Abup móvel show - SP Paralela móvel - SP Abimad - SP } \\
\text { Formóbile - SP Fenavem -SP Movinter - SP } \\
\text { Movelsul - RS Salão do móvel Brasil - RS Salão de Gramado-RS } \\
\text { Mercomóveis - SC Movelpar - PR Top Móvel-CE } \\
\text { Femur-MG Movelnorte - MA Móvel Show - ES }\end{array}$ \\
\hline Feiras setoriais & Internacionais & $\begin{array}{l}\text { Isaloni - IT } \\
\text { IMM - DE } \\
\text { Maison \& Objetct -FR }\end{array}$ \\
\hline Blogs & Nacionais & $\begin{array}{l}\text { Emobile }^{11} \\
\text { Trendmovel }^{12}\end{array}$ \\
\hline Sites & Nacionais & $\begin{array}{l}\text { Radarmobile }^{13} \\
\text { Trendmovel }^{14} \\
\text { Schattdecor }\end{array}$ \\
\hline Livros & $\begin{array}{l}\text { Nacionais e } \\
\text { internacionais }\end{array}$ & $\begin{array}{l}\text { Manual de Coolhunting Métodos e Práticas - Guia Prático para se } \\
\text { tornar um grande observador do Zeitgeist - Fah Maioli } \\
\text { O desenvolvimento de produtos sustentáveis - Ezio Manzini, Carlo } \\
\text { Vezzoli }\end{array}$ \\
\hline $\begin{array}{l}\text { Entidades, } \\
\text { Sindicatos e } \\
\text { Empresas. }\end{array}$ & Nacionais & $\begin{array}{l}\text { Cadernos ou referenciais - SENAI } \\
\text { Portal de Tendências - SENAI } \\
\text { Biomóvel - SINDUSMÓVEL } \\
\text { Schattdecor Trends }\end{array}$ \\
\hline
\end{tabular}

Fonte: Elaborado pelos autores, com base na pesquisa realizada.

A respeito da pesquisa documental pode-se afirmar que:

Com o levantamento de referenciais foi possível observar que informações disponíveis para pesquisa não faltam no Brasil. É certo que elas não estão sistematizadas, organizadas, decodificadas, prontas para serem aplicadas. Mas, ao menos campo para pesquisa tem.(DE SOUZA, 2009, p.103).

Analisando os resultados da RBS, é possível afirmar que existe um campo de pesquisa acadêmica para o projeto de móveis no Brasil. As universidades com as principais pesquisas sobre o tema encontram-se nos estados do Paraná, São Paulo e Rio Grande do Sul, fator ligado à proximidade dos polos moveleiros. Temas voltados à produção industrial, gestão do design e meio ambiente são bastante debatidos, porém, observou-se uma lacuna para pesquisas em sustentabilidade voltadas para o futuro de projeto de mobiliário.

$\mathrm{Na}$ pesquisa documental são apresentados os institutos de pesquisas onde, foram identificados 4 referências nacionais e 10 referências internacionais (Quadros 1 e 2). Essas organizações definem seus colaboradores como multidisciplinares, analisam as tendências de longo prazo (macrotendência) e disponibilizam alguns artigos resultantes de pesquisas. Porém, não deixam claro como utilizam as ferramentas de pesquisa para a obtenção de uma conclusão.

Se tratando de diretrizes projetuais orientadas por tendências, pode-se dizer que as feiras setoriais são importantes núcleos de levantamentos de dados, ajudam

\footnotetext{
${ }^{11}$ Disponível em: < http://www.emobile.com.br/site/blogs/> Acesso em Abr. 2014

${ }^{12}$ Disponível em: < http://www.trendmovel.blogspot.com.br/> Acesso em Abr. 2014

${ }^{13}$ Disponível em: < http://www.radarmobile.com.br/> Acesso em Abr. 2014

${ }^{14}$ Disponível em: < http://www.trendmovel.com//> Acesso em Abr. 2014
} 
aos visitantes identificarem novos materiais, novos fornecedores e novos parceiros para viabilizar projetos (DE SOUZA, 2009). Neste sentido, foram identificadas 15 feiras nacionais e 3 feiras internacionais que acontecem anualmente, entretanto, á indução á cópia dos produtos expostos e a resistência a novos conceitos são pontos fracos que se destacam neste evento.

Os cadernos de tendências assim como os sites, blogs, livros e cartilhas são importantes fontes de referências e inspirações para o desenvolvimento de projetos de mobiliário que apoiam empresários, principalmente os de pequenos porte, oferecendo informações sempre atualizadas que podem ser transformadas em requisitos projetuais (DE SOUZA,2009).

Estas fontes identificadas poderão fornecer informações sobre tendências de curto prazo e de longo prazo, trazendo evidencias sobre cores, formas, padrões, conceitos, combinações com componentes, outros produtos ou acessórios, novas tecnologias, materiais, etc.

Neste contexto, acredita-se que a pesquisa de tendências pode produzir o levantamento de dados necessários para o desenvolvimento de novos projetos de mobiliário. Resultando em produtos mais competitivos e inovadores alinhados com a realidade do mercado e com as necessidades dos consumidores.

\section{CONSIDERAÇÕES FINAIS}

Este artigo teve como motivação a investigação de referenciais em pesquisa de tendências para o projeto de móveis ligados a sustentabilidade.

A investigação foi realizada através da Revisão Bibliográfica Sistemática e da pesquisa documental onde foram analisados conteúdos publicados nos últimos 10 anos por meio dos portais de busca da Coordenação de Aperfeiçoamento de Pessoal de Nível Superior (CAPES) e do Google (2014).

O resultado alcançado contempla o mapeamento dos principais autores, a identificação dos campos de pesquisa acadêmica e as fontes relevantes de informações para os designers e profissionais do setor moveleiro.

Com base nos dados identificados pode-se afirmar que existe no Brasil um relativo interesse pelo estudo e pesquisa em projeto de móveis.

Conclui-se que é necessário a ampliação de novas pesquisas no meio acadêmico que tragam diferentes visões e oportunidades profissionais.

Considerando a identificação de novas variáveis, sugere-se para a realização de novos estudos: elaboração de modelos de pesquisa de tendências específicos para o setor moveleiro; estudo de ferramentas em pesquisa de tendências voltados a decodificação da sustentabilidade para projeto de mobiliário.

\section{AGRADECIMENTOS}

Os autores agradecem o apoio financeiro da CAPES (Coordenação de Aperfeiçoamento de Pessoal de Nível Superior) pela bolsa de mestrado demanda social.

\section{REFERÊNCIAS}

CETEMO. Centro Tecnológico do Mobiliário. Referências em mobiliário. Disponível na internet por http em: <http://www.cetemo.com.br/att design.html>. Acesso em 28 de Abr. de 2014. 
DE SOUZA, Aline Teixeira. DIRETRIZES PROJETUAIS E AS TENDÊNCIAS: O design de móveis residenciais no polo de arapongas/PR. 2009. 117 f. Dissertação (mestrado) Universidade Estadual Paulista "Julio de Mesquita Filho", Curso de Pós-Graduação em Design.

JAYME, Maria Emilia Mendes. TENDÊNCIAS: Recomendações para seu uso na gestão do design das MPES produtoras de louça de mesa de Campo Largo - PR. 2009. $193 \mathrm{f}$. Dissertação (mestrado) - Universidade Federal do Paraná, Curso de Pós-Graduação Mestrado em Design.

SCHATTDECOR. Disponível na internet por http em: $<$ http://www.schattdecor.de/pt/padroes-tendencias/tendencias/>. Acesso em 07 Maio. 2014.

SCHATTDECOR. Trends: Schattdecor 2012/13. Thansau (DE), 2012.

SINDUSMOBIL. Sindicado das Indústrias da construção e do mobiliário. BIOMÓVEL: sua casa mais saudável. Disponível em: <http:// www.sindusmobil.com.br/index.php/biomovel> Acesso em Abr. 2014. 